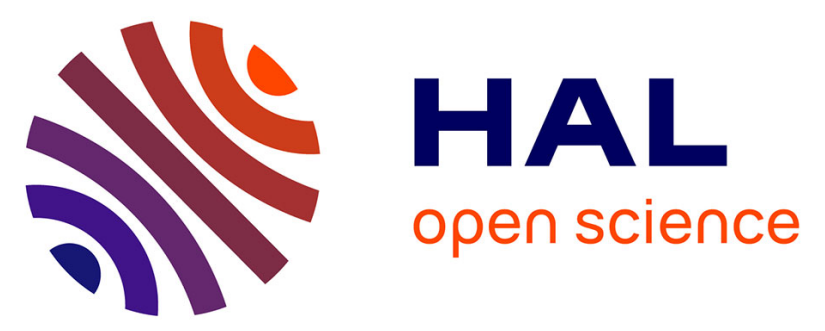

\title{
Spreading of an Oil-in-Water Emulsion on a Glass Plate: Phase Inversion and Pattern Formation
}

\author{
Antoine Deblais, Rim Harich, Daniel Bonn, Annie Colin, Hamid Kellay
}

\section{To cite this version:}

Antoine Deblais, Rim Harich, Daniel Bonn, Annie Colin, Hamid Kellay. Spreading of an Oil-inWater Emulsion on a Glass Plate: Phase Inversion and Pattern Formation. Langmuir, 2015, 31 (22), pp.5971-5981. 10.1021/la504639q. hal-01171325

\section{HAL Id: hal-01171325 \\ https://hal.science/hal-01171325}

Submitted on 15 Dec 2017

HAL is a multi-disciplinary open access archive for the deposit and dissemination of scientific research documents, whether they are published or not. The documents may come from teaching and research institutions in France or abroad, or from public or private research centers.
L'archive ouverte pluridisciplinaire HAL, est destinée au dépôt et à la diffusion de documents scientifiques de niveau recherche, publiés ou non, émanant des établissements d'enseignement et de recherche français ou étrangers, des laboratoires publics ou privés.

\section{다(1)(2)}

Distributed under a Creative Commons Attribution - ShareAlikel 4.0 International 


\title{
Spreading of an Oil-in-Water Emulsion on a Glass Plate: Phase Inversion and Pattern Formation
}

\author{
A. Deblais, ${ }^{\dagger}$ R. Harich, ${ }^{\dagger}$ D. Bonn, ${ }^{\ddagger}$ A. Colin,${ }^{\S}$ and H. Kellay ${ }^{*} \dagger$ \\ ${ }^{\dagger}$ Laboratoire Ondes et Matière d'Aquitaine, UMR 5798 CNRS U. Bx, Université de Bordeaux, 351 cours de la Libération 33405 , \\ Talence, France \\ ${ }^{\ddagger}$ van der Waals-Zeeman Institute, University of Amsterdam, Science Park 904, 1098 XH Amsterdam, Netherlands \\ ${ }^{\S}$ Centre de Recherche Paul Pascal, CNRS UPR 8641, Université de Bordeaux, 115 av. Schweitzer, F 33600 Pessac, France
}

\begin{abstract}
Rigid blade coating of glass plates by oil in water emulsions stabilized by surfactants is studied. Complete surface coverage is obtained only for speeds exceeding a threshold velocity dependent on the height between the blade end and the surface. Below this threshold, the emulsion can be inverted in the vicinity of the blade. The inversion dynamics of the oil in water emulsion and the deposition patterns induced by this phase inversion are studied using a microscope mounted set up. We show that these dynamics are universal for different volume fractions and deposition velocities. This inversion as well as the destabilization of the emulsion film deposited at high speeds gives rise to different patterns on the glass surface. These patterns are discussed in terms of the emulsion characteristics as well as the deposition velocity.
\end{abstract}

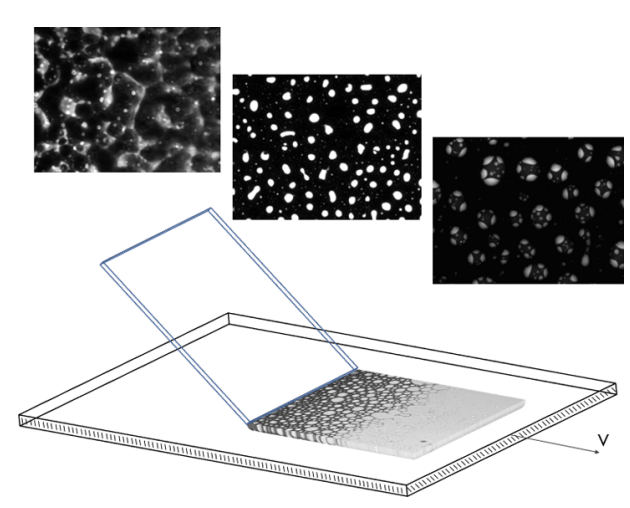

\section{INTRODUCTION}

Different techniques are used for coating solid surfaces with viscous liquids, viscoelastic fluids, pastes, or various dispersions: rigid or flexible blade coating, dip coating, and spin coating are among some of the most used methods. ${ }^{1-6}$ The stability and the thickness of the coating depend on many factors such as the viscosity and the rheology of the liquid, the blade properties, or the wetting properties of the liquid such as its contact angle with the substrate. ${ }^{6-10}$ Several studies have set the tone for understanding such coatings in Newtonian and non Newtonian fluids as the potential applications go from cleaning surfaces to coating them with functional materials to making flexible thin films. In general, the dynamics of the contact line of the fluid with the substrate, which is characteristic of all of these coating techniques, as well as the detailed nature of the flow in the immediate vicinity of the blade or in the meniscus region between the liquid and the surface play a major role but continue to pose some fundamental problems as outlined in numerous studies. ${ }^{4,11-18}$

When it comes to two fluid dispersions, much less has been done even though an emulsion is a typical class of complex fluid where different components can be enclosed; therefore, deposits with different functionalities may be obtained. The properties of the deposited film and the patterns it may give rise to are essential ingredients for making controlled coatings or flexible films, for example.

An emulsion is a concentrated dispersion of droplets of one liquid into a second one. ${ }^{19}$ Such dispersions, although metastable because the droplet sizes are in the range of micrometers, can be stabilized over long periods of time by using surface active agents. Different techniques and ap proaches are used to examine their texture and their stability. ${ }^{20}$ Emulsions have considerable industrial importance in a broad range of applications ranging from food processing to cosmetics. They may be desirable with long stability periods, but they may also be undesirable and their destruction is sought. Many factors control the stability or instability of emulsions. $^{21}$ The so called Ostwald ripening mechanism, ${ }^{19,22,23}$ which involves the diffusion of the dispersed phase through the continuous phase, is one such destabilizing factor leading to the coarsening of the droplets and to phase separation. The coalescence of droplets is another such destabilizing factor. ${ }^{19,21}$ Recent experiments have even evidenced the role of coalescence between neighboring droplets in inducing coalescence events at locations farther out. ${ }^{21}$ The destabiliza tion of an emulsion either through droplet coarsening or through coalescence between neighboring drops and its ensuing consequences necessarily lead to a phase inversion where the continuos phase becomes the dispersed phase. Under the mechanical action of say a rigid or flexible blade or even that of the meniscus in dip coating, the stability of the emulsion will be put to the test. The coating of solid surfaces with different emulsions is being considered for a variety of applications, so it is necessary to consider both the stability of these fluids under such conditions as well as the conditions for depositing a homogeneous layer. Because such emulsions will be put in contact with the solid surfaces for coating, the action of these surfaces on the stability of the emulsion will also 
be important. In sum, spreading emulsions on solid substrates will necessarily require a good knowledge of a variety of aspects. It is a subset of these considerations which will be examined here.

In this article, we describe the results of experiments on the coating of a glass substrate with an oil in water emulsion using a rigid blade as shown in the schematic of Figure 1. In these

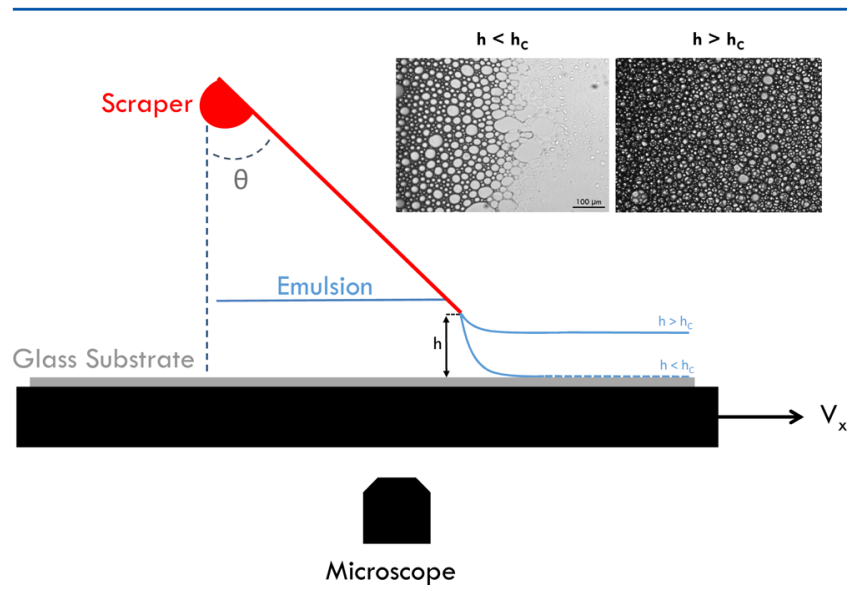

Figure 1. Schematic of the experimental setup (not to scale): It consists of a thin $(170 \mu \mathrm{m})$ microscope glass slide (of dimensions $4 \times$ $2.4 \mathrm{~cm}^{2}$ ) held fixed at an angle $\theta$ with respect to the vertical axis. The spacing $h$ between the bottom end of the scraper and the glass plate can be adjusted with a translation stage with $10 \mu \mathrm{m}$ precision. The emulsion is deposited, underneath the scraper, on a glass substrate of 6 $\times 15 \mathrm{~cm}^{2}$ in dimensions with a $2 \mathrm{~mm}$ thickness. The substrate is then translated in the $x$ direction with a micrometric motorized translation stage (Marzhauser Wetzlar SCAN IM $130 \times 100$ ) capable of moving at stable speeds ranging from $0.1 \mu \mathrm{m} / \mathrm{s}$ to $10 \mathrm{~mm} / \mathrm{s}$. This setup is mounted on a microscope stage. The two upper photographs show the result of deposition below and above the critical height $h_{\mathrm{c}}$ : no spreading below $h_{\mathrm{c}}$ with the emulsion remaining near the scraper and film spreading above $h_{\mathrm{c}}$.

experiments, the blade or scraper is held fixed while the glass substrate containing a large drop of emulsion and positioned underneath the scraper is translated at different velocities $V$. The solid blade makes an angle $\vartheta$ (which is held constant) with the substrate, and its end is positioned a distance $h$ from the plate. We first present the phase diagram for depositing a homogeneous emulsion film using this process and then discuss the different patterns obtained.

Our experiments show that homogeneous emulsion film formation on the solid surface occurs under well defined conditions in the parameter space described by the height of the blade $h$ and the velocity of the plate $V$. For a fixed velocity, film formation occurs only when the height exceeds a threshold height noted $h_{c}$. This height decreases with the translation velocity. Equivalently and for a fixed height $h$, a homogeneous emulsion film is obtained only for velocities above a threshold value $V_{c}$. In other words, for low translation velocities or low heights, the emulsion partially wets the substrate, making a well defined contact angle with the glass plate in the region near the scraper end. For high velocities or large heights, on the other hand, a homogeneous emulsion layer can be spread on the glass plate. Note that close to $h_{c}$ or $V_{c}$ the distance over which the emulsion is deposited goes from a finite value (partial spreading) to the length of the glass substrate (total spreading). This transition is reminiscent of the dynamic wetting transition of partially wetting Newtonian fluids in dip coating experi ments.

In these two situations (partial wetting of the emulsion below $h_{\mathrm{c}}$ or $V_{\mathrm{c}}$ or a homogeneous emulsion film above), the emulsion becomes unstable due to the presence of evaporation of the continuous aqueous phase either through a drying front or through a nucleation and growth mechanism, and phase inversion occurs, giving rise to an oil film with different embedded patterns depending on the composition of the emulsion. We discuss the results of the demixing dynamics and the efficiency of this system to invert the emulsion. The patterns that result from the destabilization and inversion of the emulsion go from simple water drops and multiple drops embedded in an oil film to more complex interconnected and percolated patterns. These patterns are discussed in light of the surfactant used and the affinity of the ingredients with the surface. Our experiments suggest that this simple system may be useful for studying emulsion film formation, emulsion inversion, and pattern formation under a variety of conditions.

\section{EXPERIMENTAL SECTION}

Our experiments were performed on emulsions consisting of oil droplets dispersed in water and stabilized by surfactant. Two different emulsions were used: ${ }^{24,25}$ one consisting of castor oil (Sigma Aldrich, viscosity $1000 \mathrm{cst}$ ) and the ionic surfactant SDS (sodium dodecyl sulfate, Sigma Aldrich) and another consisting of silicone oil (Sigma Aldrich, viscosity $1000 \mathrm{cst}$ ) and nonionic surfactant NP7 (Tergitol, Sigma Aldrich). The continuous phase for the first emulsion consists of pure water and SDS at either a 2 or $1 \%$ mass fraction while it consists of pure water and NP7 at a $3 \%$ mass fraction. The emulsions are prepared using the viscous phase method which allows us to obtain different mean drop diameters (between 3 and $10 \mu \mathrm{m}$ ) and different polydispersity ratios (the mixers used are IKA T18 and IKA Eurostar 60 ). We slowly add oil to the continuous phase while mixing with an emulsifier up to a volume fraction $\Phi$ of $85 \%$. For certain experiments higher surfactant concentrations were also considered, and glycerol (from Sigma Aldrich) was added to the continuous phase in different proportions in some cases. We obtained samples with lower $\Phi$ by dilution of the stock emulsion with the continuous phase. The rheological properties of the emulsions used have been studied before ${ }^{24}$ with the result that the flow curves can be described by the so called Herschel Bulkley model $\sigma=\sigma_{y}+k \dot{\gamma}^{n}$, where $\sigma$ is the stress, $\dot{\gamma}$ is the shear rate, $\sigma_{y}$ is the shear yield stress, $k$ is the consistency parameter, and $n$ is the index of shear thinning taking values near 0.5 . The yield stress generally depends on the volume fraction and increases as this fraction increases. Our own measurements using a rough plate geometry on an ARG2 controlled stress rheometer confirm that the emulsions used here exhibit similar behavior, allowing us to determine $\sigma_{y}, k$, and $n$ for each system. These values are given in Table 1.

The surface tensions of the different fluids against air were determined using the drop weight method or a spinning drop tensiometer. The contact angles with the glass substrate used were determined using photographs of small drops deposited on the glass plate. A summary of this data is given in Table 1. Note that on the glass plate used, both emulsions partially wet the substrate and do not spread. No particular surface treatment was used for the glass plate which was cleaned using a soap solution first, rinsed with pure water, and cleaned using ethanol before drying.

To spread the emulsions on the glass substrate, a simple homemade setup was designed and mounted on a microscope stage for observation as shown in the schematic of Figure 1. The mechanical tool used for depositing the emulsion on the glass substrate (thickness $2 \mathrm{~mm}$, width $6 \mathrm{~cm}$, and length $15 \mathrm{~cm}$ ) consists of a rigid blade and a thin rectangular microscope glass slide of $170 \mu \mathrm{m}$ thickness $(2.4 \mathrm{~cm}$ width and $4 \mathrm{~cm}$ length) whose bottom end is held at the desired distance $h$ from the glass substrate on which the emulsion is to be 
Table 1. Parameters of the HB Model for the Fluids Used and the Contact Angles of the Different Constituents of the Emulsions Used with the Glass Substrate

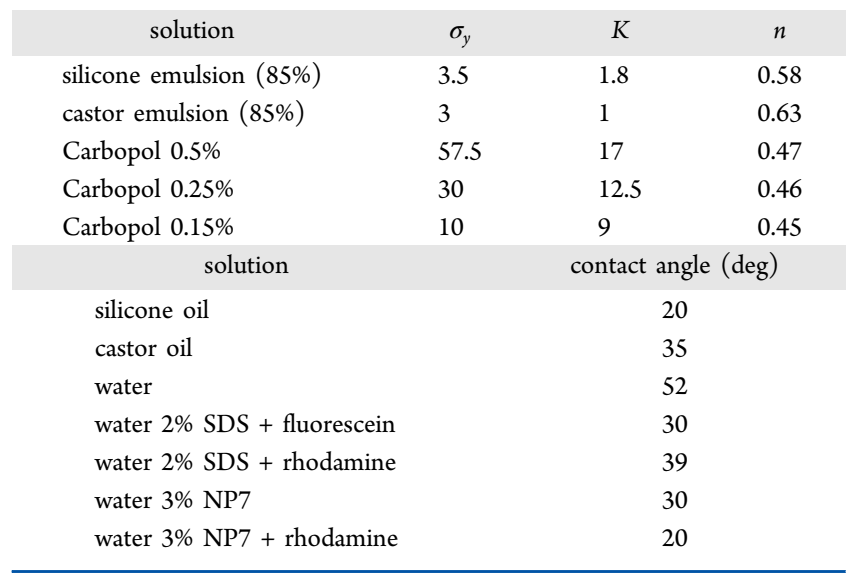

deposited. The distance $h$ between the bottom end of the blade and the glass substrate can be set using a precision translation stage and was varied from about $500 \mu \mathrm{m}$ down to $20 \mu \mathrm{m}$. The angle of the blade with respect to the vertical direction $\vartheta$ can also be adjusted between 15 and $90^{\circ}$. Most of the experiments presented here are for $\vartheta=45^{\circ}$.

This setup is assembled on an inverted microscope stage and allows us to study the in situ deposition or destabilization of the emulsion on the glass substrate. We use a microscope (Zeiss Axio observer A1) and a fast camera (Hamamatsu Orca flash 2.8 or a phantom V641) to record the spreading of the emulsions either in transmission in bright field imaging or by reflection using fluorescent molecules fluorescein or rhodamine (from Sigma Aldrich and Exciton) in the continuous water phase. Rhodamine is excited at $500 \mathrm{~nm}$ and emits at $540 \mathrm{~nm}$ whereas fluorescein is excited at $525 \mathrm{~nm}$ and emits at $550 \mathrm{~nm}$.
Fluorescence imaging allows us to distinguish between the dispersed and the continuous phases in the deposited films.

The glass substrate was held on a motorized precision translation stage capable of translation velocities of between $0.1 \mu \mathrm{m} / \mathrm{s}$ and 10 $\mathrm{mm} / \mathrm{s}$. The emulsion was deposited behind the glass scraper as a large, thick drop, and the translation of the motorized stage allows us to entrain the emulsion through the opening between the scraper end and the glass substrate and eventually deposit the emulsion at different speeds. Most of the experiments were carried out at humidity rates in the range 30 to $50 \%$, so evaporation of the continuous aqueous phase was always present. A few experiments were carried out at higher rates near $85 \%$ to limit the effects of evaporation.

\section{RESULTS}

A. Emulsion Spreading: From Partial to Complete Spreading. First, we focus on the properties of the deposited layer versus the height $h$ of the blade and the velocity of the bottom plate $V$. In Figure $2 A, B$ we show the results of this characterization for the two emulsion systems used. As mentioned above, spreading a homogeneous layer of the emulsion occurs, for a fixed velocity $V$, only when $h$ is greater than a height $h_{\mathrm{c}}$ or, equivalently, for a fixed height $h$ only when the velocity increases above a threshold $V_{c}$. The locus of points $h_{\mathrm{c}}$ and $V_{\mathrm{c}}$ is, roughly, a straight line, $h_{\mathrm{c}}=a\left(V-V_{\mathrm{c}}\right)$, as indicated in Figure 2 for the two systems. Parameters $a(1.3$ and $0.9 \mathrm{~s}$ in Figure $2 \mathrm{a}, \mathrm{b}$, respectively) and $V_{\mathrm{c}}(340$ and $350 \mu \mathrm{m} / \mathrm{s}$ in Figure $2 \mathrm{a}, \mathrm{b}$, respectively) are system dependent as seen in Figure 2. Above the critical height $h_{c}$ and the critical velocity $V_{\mathcal{c}}$ a homogeneous layer of the emulsion can be deposited on the glass plate as seen in the photographs of Figure 2. Below $h_{c}$ or $V_{\mathcal{c}}$ the emulsion remains near the blade and forms a meniscus in the vicinity of the scraper. The images in Figure 2 show this region for the two emulsions studied. For a fixed height, the

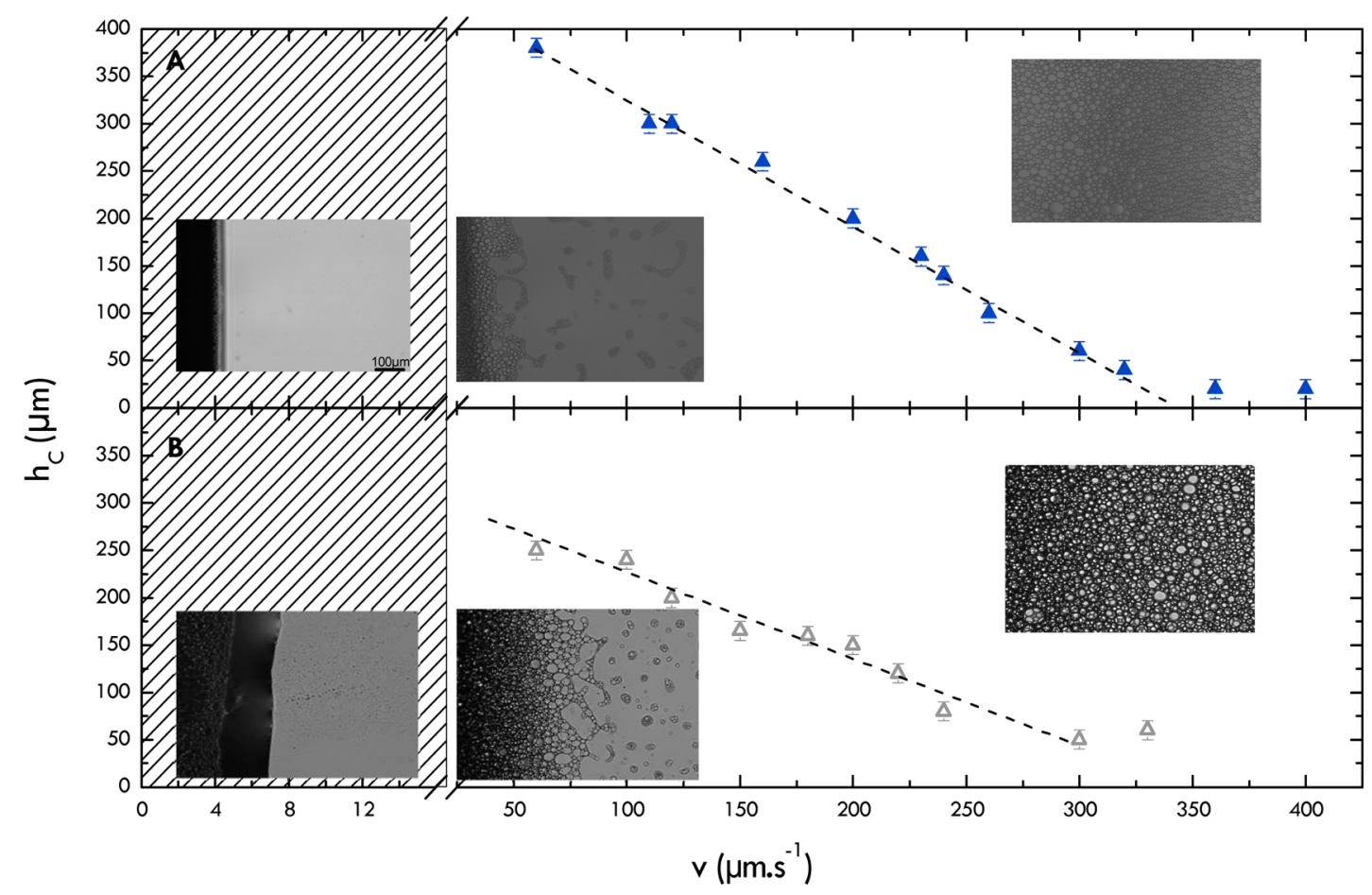

Figure 2. Spreading phase diagrams: $h_{c}$ versus the spreading velocity $V$. For each velocity, if the distance between the scraper and the substrate is greater than $h_{\mathcal{c}}$, then a homogeneous layer of the emulsion can be deposited. For lower heights, either water drops or demixing of the emulsion occurs. Representative photographs of the deposited layer are shown for different heights and speeds. Left to right: $V=13 \mu \mathrm{m} / \mathrm{s}$ and $h=10 \mu \mathrm{m}, V=$ $60 \mu \mathrm{m} / \mathrm{s}$ and $h=10 \mu \mathrm{m}, V=200 \mu \mathrm{m} / \mathrm{s}$ (for upper image), $V=180 \mu \mathrm{m} / \mathrm{s}$ (for lower image) and $h=300 \mu \mathrm{m}$. (A) Silicone oil in water emulsion and (B) castor oil in water emulsion, both at a volume fraction of $85 \%$. 
distance between the blade and this meniscus region increases as the velocity increases; at $V_{\mathrm{c}}$ and above, the formation of a homogeneous film occurs and no meniscus is visible as confirmed by visualizing the glass plate under the microscope at different distances from the scraper. This phenomenology is analogous to the dynamic wetting transition which occurs in dip coating experiments for partially wetting fluids, which is the case for our emulsions which partially wet the glass plate. However, the transition to homogeneous film deposition or dynamic wetting seems very different from that of a viscous Newtonian fluid. ${ }^{14,15,18}$ For a nonwetting Newtonian fluid and as the velocity of the plate increases in dip coating experiments, the contact angle decreases and vanishes at a relatively well defined velocity above which a film is deposited. This transition is generally reported versus the so called capillary number $\mathrm{Ca}=$ $\eta V / \gamma$, where $\eta$ is the viscosity of the liquid and $\gamma$ is the surface tension of the fluid. The dynamic wetting transition is found for capillary numbers of between $5 \times 10^{-3}$ and $9 \times 10^{-3}$ and depends on the contact angle of the fluid at rest. In our case, for each height of the blade, a threshold velocity $V_{c}$ is needed for the transition to occur. If one were to construct a critical capillary number for this transition, then this number will depend on the height of the blade even if the variation of the viscosity with shear rate ${ }^{24}$ (estimated using $V_{c} / h_{c}$ as the relevant shear rate) is taken into account. The transition occurs at capillary numbers that vary from roughly $50 \times 10^{-3}$ at velocities near $50 \mu \mathrm{m} / \mathrm{s}$ down to $10 \times 10^{-3}$ at velocities near $400 \mu \mathrm{m} / \mathrm{s}$ for the castor oil emulsion. This is shown in Figure 3 for the

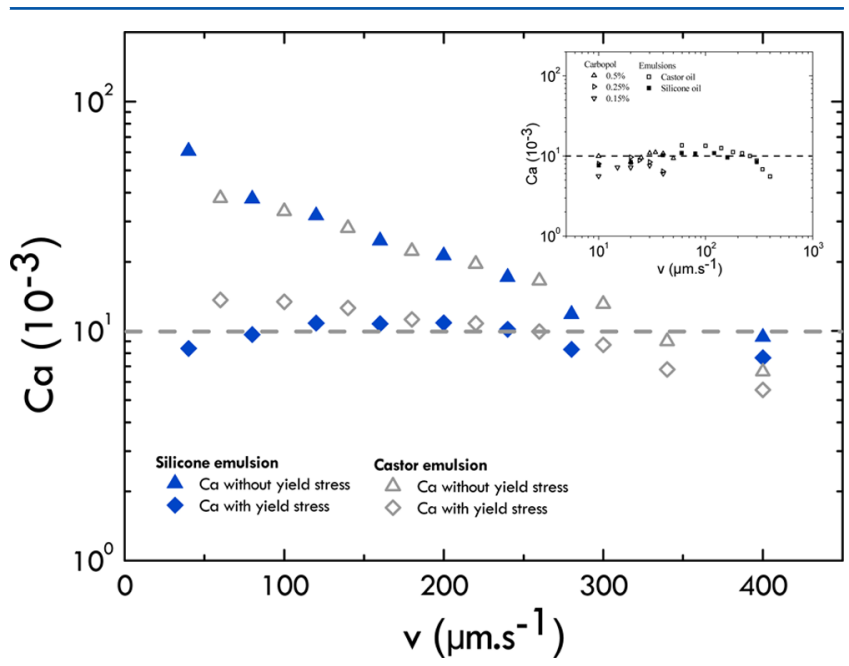

Figure 3. Capillary number for the transition to film formation versus plate velocity. The capillary numbers shown are for the two different systems and with or without taking the yield stress into account. The inset shows similar data along with transition capillary numbers obtained using Carbopol dispersions at different concentrations and therefore different rheologies.

two systems used. These transition capillary numbers are larger than those of Newtonian fluids in dip coating experiments for example, ${ }^{14}$ except at high velocities. A better transition criterion can be obtained by redefining the viscous stress and taking into account the fact that these fluids have a yield stress. Let us redefine the transition capillary number $\mathrm{Ca}$ as $\left(\sigma-\sigma_{y}\right) /\left(\gamma / h_{\mathrm{c}}\right)$. The rationale behind this redefinition is that for the fluid to flow, the applied viscous stress $\sigma$ needs to be larger than the fluid yield stress $\sigma_{y}$. The viscous stress is then weighted by the capillary pressure in the meniscus region where a typical length scale is $h$. To use this definition, we estimate the shear rate $\gamma$ at the transition as $V_{\mathrm{c}} / h_{\mathrm{c}}$ first, and using the flow curves for the emulsion (obtained using a rheometer), we obtain the applied stress $\sigma$. The yield stress $\sigma_{y}$ is also obtained from the fluid flow curves. This definition of the critical capillary number gives roughly constant values near $10 \times 10^{-3}$ for all of the velocities examined between 50 and $400 \mu \mathrm{m} / \mathrm{s}$ and for the two systems used (the surface tensions used are $20 \mathrm{mN} / \mathrm{m}$ for the castor oil emulsion and $20 \mathrm{mN} / \mathrm{m}$ for the silicone oil emulsion).

This criterion for the transition to film deposition of yield stress fluids can be tested using other yield stress fluids. We have used Carbopol suspensions at different concentrations to vary the rheological properties of the fluid. We have carried out similar spreading experiments as for the emulsions studied above and found similar behavior. In order to form a homogeneous film of the Carbopol solutions, a critical height and a critical velocity are needed for spreading to occur. The capillary numbers for the transition are reported in the inset of Figure 3 along with the data from the two emulsion systems. Here again, the use of a redefined capillary number seems to indicate that the transition occurs at a roughly constant capillary number as for the emulsions used. While the data obtained do not show a perfect plateau for the transition capillary number, the variation of this number turns out to be much smaller than using the usual definition of $\mathrm{Ca}$ which does not take the yield stress into account. Also, the Carbopol solutions seem to give a transition at a slightly smaller capillary number than the emulsions, but this is probably due to differences in contact angles between the two systems.

A simple redefinition of the capillary number seems to give a relatively well defined criterion for the dynamic wetting transition observed here for yield stress fluids; however, it is possible that other effects need to be taken into account to fully understand this phenomenology. A possibility is that to spread the emulsion as a thin homogeneous film, the drops need to deform to enter the meniscus region formed by the continuous phase with the substrate. Recent work has shown that additional features related to the deformation of the drops through a capillary contribution need to be taken into account. ${ }^{26}$ However, including such a phenomenology in the transition from partial to complete wetting in the case where a large number of drops is present remains to be done. Also, for certain yield stress fluids, large slip velocities may occur near the scraper end, and our redefinition of the capillary number does not take this into account. The region at very small velocities (the dashed region in Figure 2) for which no critical height could be found is probably a signature of this slip at the surface. Measurements of the velocity profile under the scraper show that our estimate of the shear rate is slightly higher (by about $10 \%$ ) than what is measured, again indicating the possibility of slip, but the error in the estimated shear rate remains small. Another possibility is that the apparent yield stress may depend on the height of the blade, ${ }^{27}$ especially when this height is small (or comparable to emulsion drop dimensions), and this effect may influence the transition. Nonetheless, the fact that a simple redefinition of the capillary number gives reasonably constant values for the critical capillary number for the transition may indicate that such effects are small and probably within the precision of our experiments.

B. Meniscus Region and Emulsion Inversion below the Spreading Transition. Besides the spreading criterion, discussed above, which is different from the simple Newtonian 
case, the meniscus region formed by the emulsions near the scraper for heights and velocities smaller than $h_{\mathrm{c}}$ and $V_{\mathrm{c}}$ exhibits complex dynamics. For very small velocities (the dashed region in Figure 2), a clear meniscus region delimiting the emulsion from the substrate is clearly visible in the photographs of Figure 2 , but small drops of the continuous phase (water) are entrained from this region by the translation of the substrate. For intermediate velocities below the spreading transition, the photographs in Figure 2 and 4 show that while the contact line

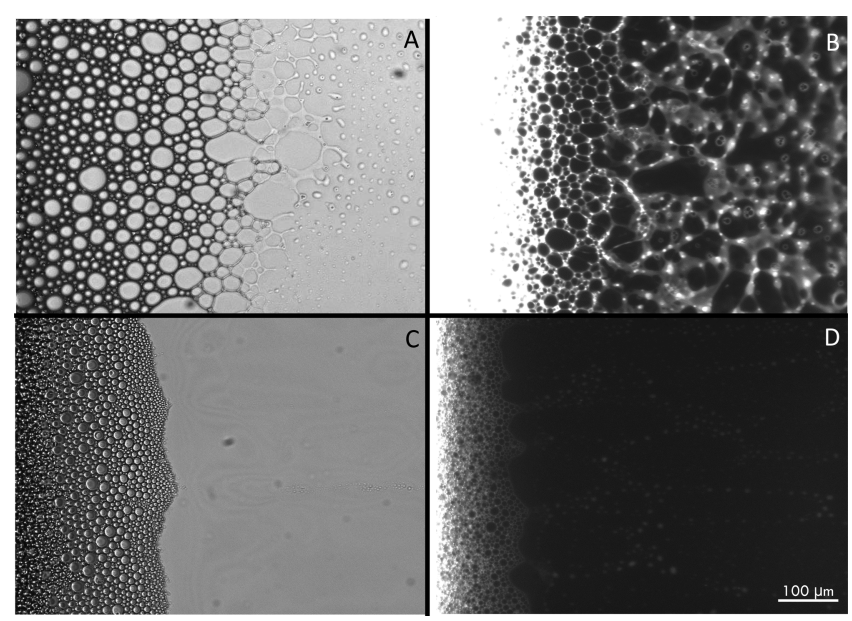

Figure 4. Observation (downstream of the scraper) of emulsions deposited on a glass substrate for $h<h_{c}$. Photographs A and B are for the castor oil emulsion, and $\mathrm{C}$ and $\mathrm{D}$ are for the silicone oil emulsions. (A) Emulsion break up at a well defined front where oil droplets coalesce and give rise to an oil layer. The continuous water phase is visible as either droplets or elongated ligaments. Image B shows the same type of front but in fluorescence imaging where water appears bright and oil is dark. Images $\mathrm{C}$ and $\mathrm{D}$ are for the silicone oil emulsion. Note that whereas the front is visible its properties are different: there is no visible coalescence between droplets (which actually merge with the substrate). Oil is still deposited on the substrate, with water drops clearly visible in the fluorescence imaging shown in D.

remains well defined and clearly visible for the silicone oil system, the castor oil droplets get deformed and coalesce with each other, leading to a destabilization and inversion of the emulsion in the meniscus region. Despite the quiescent appearance of the contact line region of the silicone oil emulsion, a closer look shown in Figure 5 indicates that drops may disappear by coalescence with the substrate. Both emulsion systems are therefore unstable near the contact line, giving rise to a complex meniscus. The destabilization of the emulsion in this region is not the same for the two emulsion systems. The castor oil system is destabilized by the deformation of drops and the coalescence of neighboring drops in a thin emulsion layer. The silicone oil system is destabilized by the coalescence of drops with the glass substrate. The two different mechanisms lead to the formation of an oil film containing the continuous phase in the form of small droplets or more complex structures as seen in Figure 4 for the two different systems. Both emulsion systems undergo a phase inversion in this meniscus region where the dispersed phase (oil) forms a layer on the substrate and the aqueous phase forms dispersed drops or patterns. The patterns for the castor oil emulsion turn out to be more complex as compared to the patterns formed by the destabilization and inversion of the silicone oil emulsion. Fluorescent images of this layer are shown in Figure 4, where small water drops are clearly visible for the silicone oil system while an intertwined printed network of water ligaments characterizes the castor oil system.

Clearly, the two systems do not behave similarly with respect to the destabilization and eventual inversion of the emulsion near the contact line. The differences between the two systems can be observed clearly in Figure 5 where for the silicone oil system drops seem to disappear without any coalescence with their neighbors. Once a few drops have disappeared from the imaged area, a spot devoid of emulsion appears. This spot increases in size rapidly. We believe that this spot is made of oil which wets the glass substrate, causing the water based emulsion to dewet. On the contrary and for the castor oil emulsion, the drops grow in size mainly by coalescence with neighboring drops, leading to an eventual breakup of the large drops to end up forming an oil film on the substrate.

In the case of silicone oil emulsions, the water ends up as small drops in the oil film (Figure 4) whereas for the castor oil emulsion, the water is in the form of a network of ligaments connected to each other. This network eventually relaxes to give rise to large patches of water in the form of films and drops.

The differences between the two systems are due to different factors: the water/castor oil surface tension is lower than that for the water/silicone oil interface $(0.55$ and $1.65 \mathrm{mN} / \mathrm{m}$ respectively), allowing the drops to deform more easily for the castor oil and be entrained in the thin layer near the meniscus region. ${ }^{26}$ Also, the wetting of the silicone oil on the glass substrate is more favorable, leading the emulsion oil drops to coalesce with the glass. The measured contact angles are given in Table 1 and show that the contact angle of the silicone oil $\left(20^{\circ}\right)$ is smaller than that of water $\left(52^{\circ}\right)$ or water with additives. The meniscus region for emulsions is therefore host to complex dynamics leading to the deposition of the constituent phases (oil or water) well below the transition to homogeneous emulsion film formation.

C. Coalescence Dynamics for the Castor Oil Emulsion. As outlined above, the emulsion inverts in the meniscus region for both systems under study. In general, emulsion inversion may occur following different scenarios. An emulsion will generally lose its stability when the volume fraction of the dispersed phase increases above a threshold value known empirically (roughly 90\%). In a recent paper, a drying stress was found to lead to phase inversion via a coalescence front or through a nucleation and growth mechanism. ${ }^{29}$ Drying in this case increases the local volume fraction and leads to the coalescence of droplets. In our case and below $h_{\mathcal{c}}$ not only is the castor oil emulsion confined in the meniscus region, causing the drops to deform due to their low surface tension, but also evaporation of the continuous phase in the thin layer near the meniscus may cause the surface fraction of the drops to increase, leading to emulsion inversion. We have tested the role of evaporation through a change in the humidity in a few experiments and noted that when the humidity rate is large enough (typically above $85 \%$ ) the front was much less prone to destabilization, indicating that evaporation is playing an important role. However, we did not disentangle the role of meniscus deformation from the role of humidity.

As mentioned above, both emulsions undergo an inversion in the vicinity of the blade near the contact line between the emulsion and the glass substrate. In general, the drops enter this meniscus region, which becomes thinner and thinner as the distance from the scraper increases (fluorescence intensity 


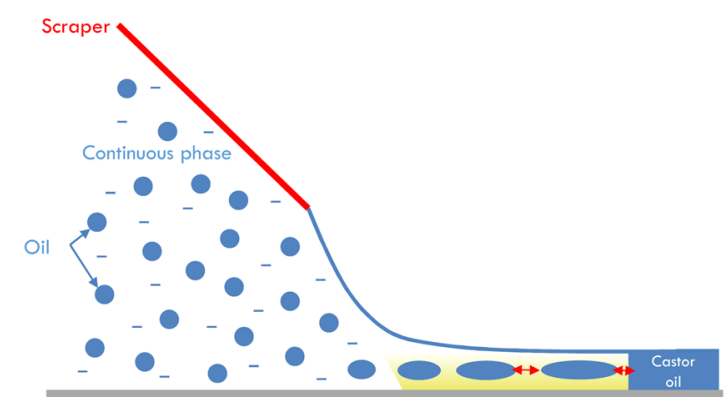

A
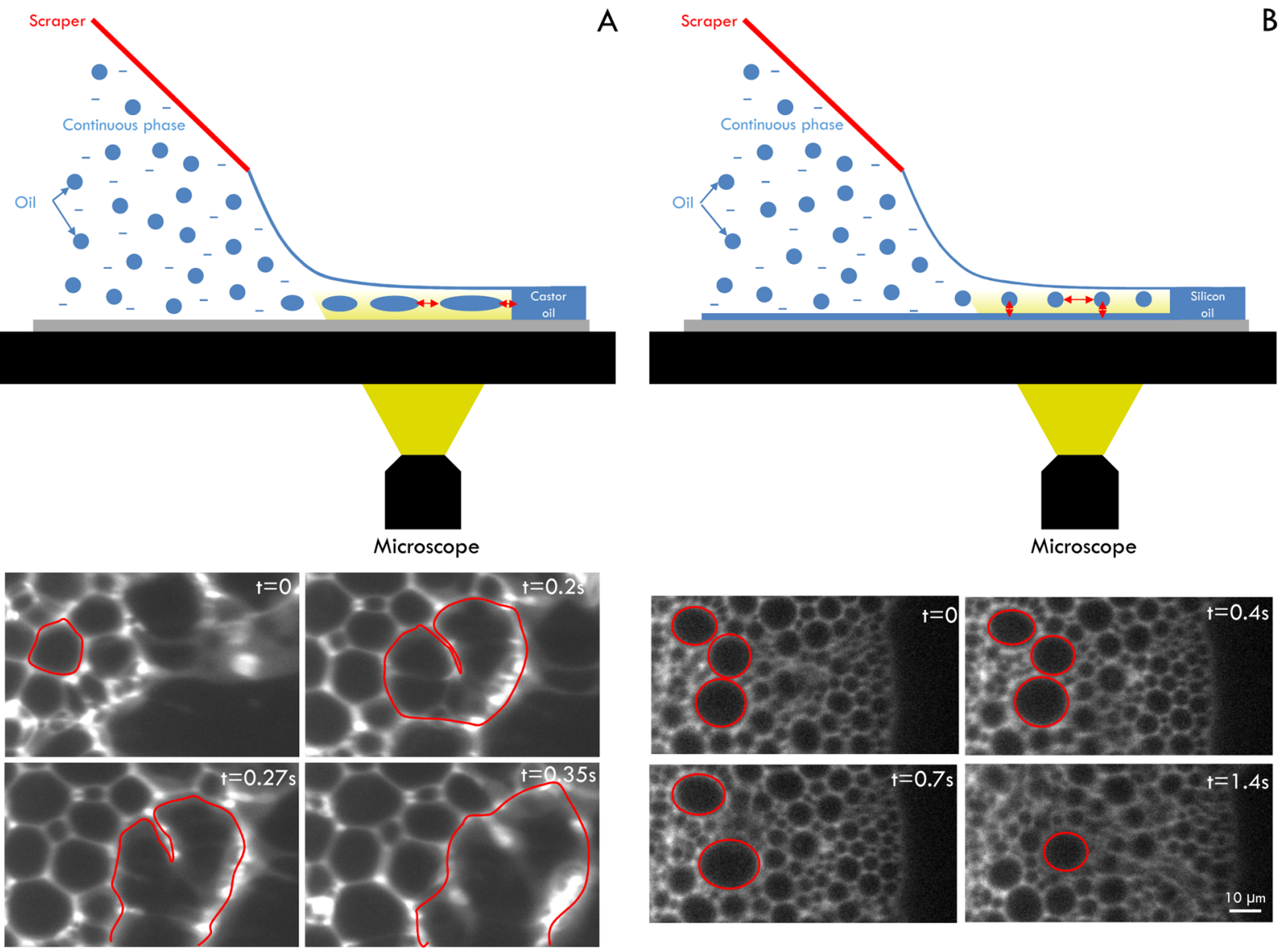

Figure 5. Destabilization of the emulsions. (A) Contrary to the silicone oil emulsion, the castor oil drops coalesce with each other to form larger droplets which end up breaking to form an oil deposit on the substrate. (B) Silicone oil emulsion. Note that certain drops in these photographs taken using fluorescence imaging (the water appears bright and the oil dark) simply disappear from view as they coalesce with the bottom glass plate. In a later stage, the emulsion ends up dewetting the substrate because oil has been deposited on the surface. Schematic view of the destabilization mechanism for each emulsion with lateral drop coalescence for the castor emulsion and the disappearance of drops through coalescence with the glass slide for the silicone system.

measurements indicate a decrease of the intensity versus distance from the scraper, indicating the presence of a thickness profile), they flatten out and get deformed. This deformation gives an apparent drop size increase. Then, these deformed drops either coalesce with each other or with the glass substrate depending on the type of emulsion used. In the first case, the drop area increases rapidly in time before the drops merge with the oil film downstream whereas in the second case no apparent increase is observed.

Let us now focus on the coalescence of drops with each other in the castor oil case and examine how the apparent size of the drops increases in time, leading to an inversion of the emulsion. Note that this cannot be carried out for the silicone oil emulsions as no coalescence is observed in this case. To gauge how deformation and coalescence lead to phase inversion, we measured the apparent area of the drops as they get entrained away from the deposition zone. The tracking of individual oil droplets from near the scraper end to the demixing front shows that the droplet size grows slowly at first with no apparent coalescence with neighboring drops: this growth is probably due to the deformation of the drops as they enter the thin meniscus region. We cannot exclude at present coalescence with smaller drops which are not easily visible. Coalescence between neighboring drops then clearly occurs in a second region farther upstream. This coalescence ends up inverting the emulsion completely. The initial growth of the projected drop area appears to be roughly linear at first as shown in Figure 6A (upper panel) and the inset of Figure 6A, but then the growth is more rapid, leading to a larger increase in the drop size in a finite time before merging with the oil film upstream. Examples from several drops are shown in Figure 6A (inset). Note that drops of different initial sizes show roughly similar growth. Also, different deposition velocities give rise to roughly similar growth dynamics. To analyze how this growth proceeds in time, we have carried out two different analyses. In the first analysis, we normalize the drop size by the initial drop area. In order to proceed further, we average, for each deposition velocity, the dynamics from a large number of drops. Now and in order to collapse data for different deposition velocities, we normalize by a characteristic time $\tau$ (shown in the inset of Figure 6A) for each velocity (roughly the transit time from scraper to front end), which amounts to setting a similar initial growth rate for all of the runs. This then collapses all of the growth dynamics for different velocities and different volume fractions on a single master curve as Figure 6A illustrates.

The dynamics are well represented, at least for late times, by a functional form

$$
\frac{A(t)}{A_{0}}=\left(\frac{t_{c}-t}{\tau}\right)^{\alpha}
$$



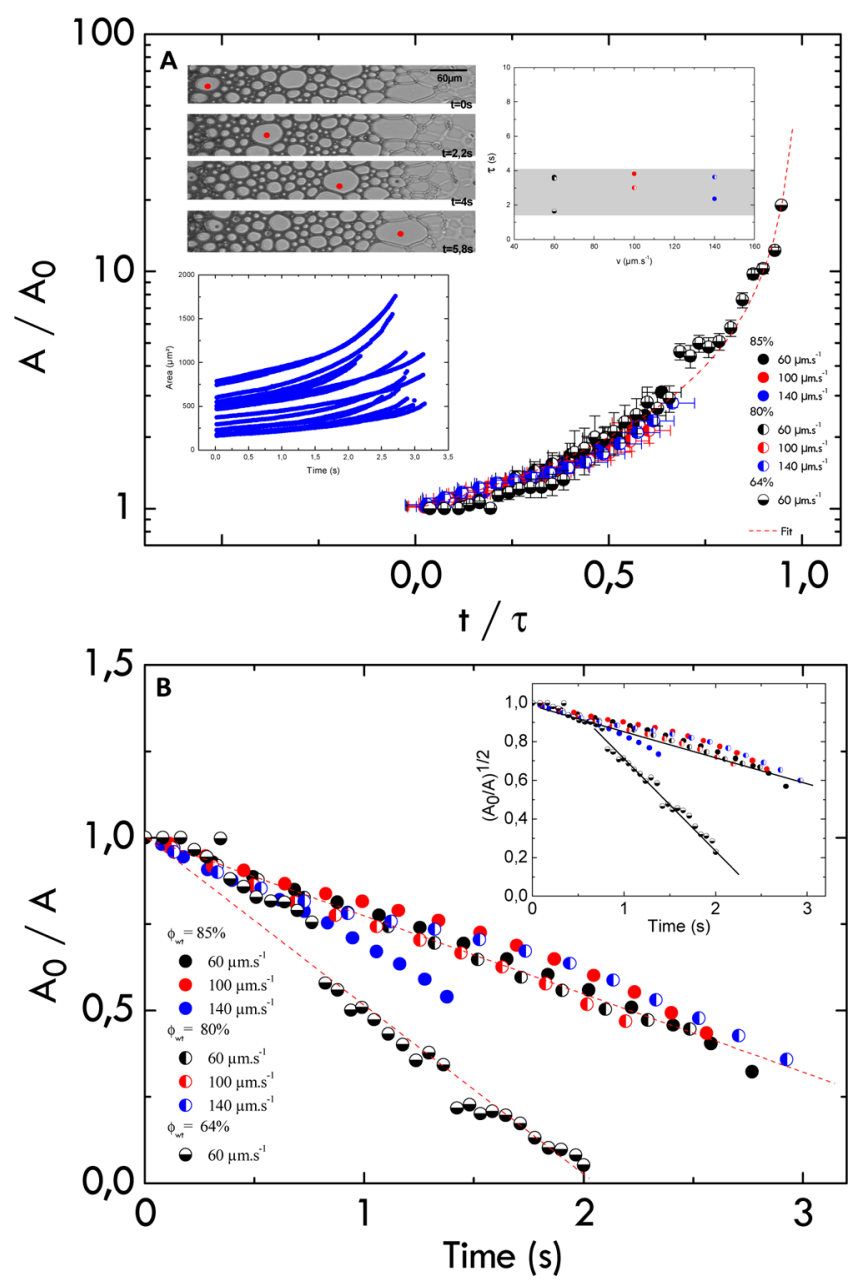

Figure 6. Dynamics of coalescence and destabilization for the castor oil emulsion for $h<h_{c^{*}}$ (A) Average droplet size normalized by the initial size versus normalized time for different spreading velocities and different volume fractions. The upper left inset shows images of the front with a marked drop undergoing an increase in size. Lower left inset: different drop sizes are represented for a single translation velocity and volume fraction. The main figure shows the average evolution obtained from averaging the dynamics of several drops. The time $\tau$ turns out to be a few seconds (see inset) and is of the same order of magnitude as the transit time of the drop as it is advected from near the scraper to the demixing front. The red line represents a fit to the data according to the functional form for A (eq 1) following a power law and given in the main text. (B) Different analysis of the data using the inverse of the drop size normalized by the initial size versus time. Different volume fractions and velocities are used. The line indicates a linear decrease versus time as expected theoretically (eq 2). The inset shows an analysis for a two dimensional layer (eq 3).

where $A(t)$ is the projected drop area and $A_{0}$ is the initial drop area. Here $t_{c}$ is the estimated time for which the drop size becomes infinite as the drop joins the oil layer downstream and is given by $t_{\mathrm{c}} \simeq \tau$. The exponent $\alpha=-1$ gives a reasonable fit to the data shown in Figure 6A where different volume fractions and different deposition velocities are used. The inversion of the emulsion proceeding via droplet coalescence seems to undergo a scaling reminiscent of finite time singularities according to this analysis. The exact reasons for this scaling are not clear to us at present. However, a study of emulsion drop coarsening in monodisperse emulsions ${ }^{28}$ does indicate that the drop diameter $D$ grows in time as

$$
\frac{1}{D^{2}}=\frac{1}{D_{0}^{2}}-C \omega t
$$

where $D_{0}$ is an initial diameter, $t$ stands for time, $\omega$ is a frequency of coalescence per unit surface area, and $C$ is a numerical constant. This model therefore shows that drop size may diverge at a finite time. The value of $\alpha=-1$ that we find above is consistent with the proposed linear scaling. A variant of this model for our case may consider a coalescence frequency per unit length instead of unit surface if we assume that our drops are actually thin disks as they are flattened in the meniscus region. The scaling of the growth of the diameter in this case would read

$$
\frac{1}{D}=\frac{1}{D_{0}}-C \omega t
$$

Although our data are not extensive enough to distinguish between the two scalings as shown in Figure 6B and its inset, the late time dynamics may be consistent with this latter scaling. Note that a test of these two scalings is presented in Figure 6B: if we identify $A$ with $D^{2}$, then we expect $\alpha=-1$ if eq 2 is valid or $\alpha=-2$ if eq 3 is valid. The inset of Figure 6B plots $\left(A / A_{0}\right)^{1 / 2}$, so a linear dependence in this inset is indicative of the validity of eq 3 . A curious feature coming out of these two graphs is that the more dilute sample seems to follow the same variation as the more concentrated samples at first before showing a faster coalescence rate. This observation probably indicates that the early time dynamics and the late time dynamics are not the same: early times are dominated by deformation, and late times are dominated by coalescence.

D. Emulsion Destabilization and Pattern Formation below and above $\boldsymbol{h}_{\mathrm{c}}$. The meniscus region below the spreading transition is subject to evaporation, and phase separation occurs. This is also true above the transition where the homogeneous film is prone to evaporation. This emulsion layer becomes unstable (due to evaporation and eventual coalescence between drops or with the substrate). Along with this destabilization, an oil film forms on the substrate containing the continuos phase in the form of drops, ligaments, or more complex structures. Such structures are also obtained for smaller heights as well. We now describe the different patterns obtained and contrast the two different systems.

First, let us focus on the castor oil emulsion system. As mentioned above, this system is unstable through the coalescence of drops in a thin layer. This occurs both below and above $h_{c}$. Below $h_{c}$, the inversion of the emulsion occurs at a certain distance from the blade, giving rise to a demixing front which ends up engendering an intertwined pattern of water ligaments embedded in an oil film. This also occurs above $h_{\mathrm{c}}$ when the homogeneous emulsion film breaks up through the presence of a drying front propagating toward the blade and leading to an oil film containing water ligaments. The ligaments are of variable sizes and heights. In both cases, the inversion of the emulsion gives rise to an interconnected random network of water ligaments as shown in Figures $4 \mathrm{~b}$ and $7 \mathrm{~A}$ in the initial stages. This may be of practical use because it indicates that this system may give rise to a percolated network of water channels useful for depositing thin conducting films, for example. The reason behind the existence of this network is related to the wetting properties of the different components of this system: the continuous phase is more favored at the glass surface. For long times, these water ligaments spread out (Figure 7A) on the glass surface and end up giving rise to a roughly 


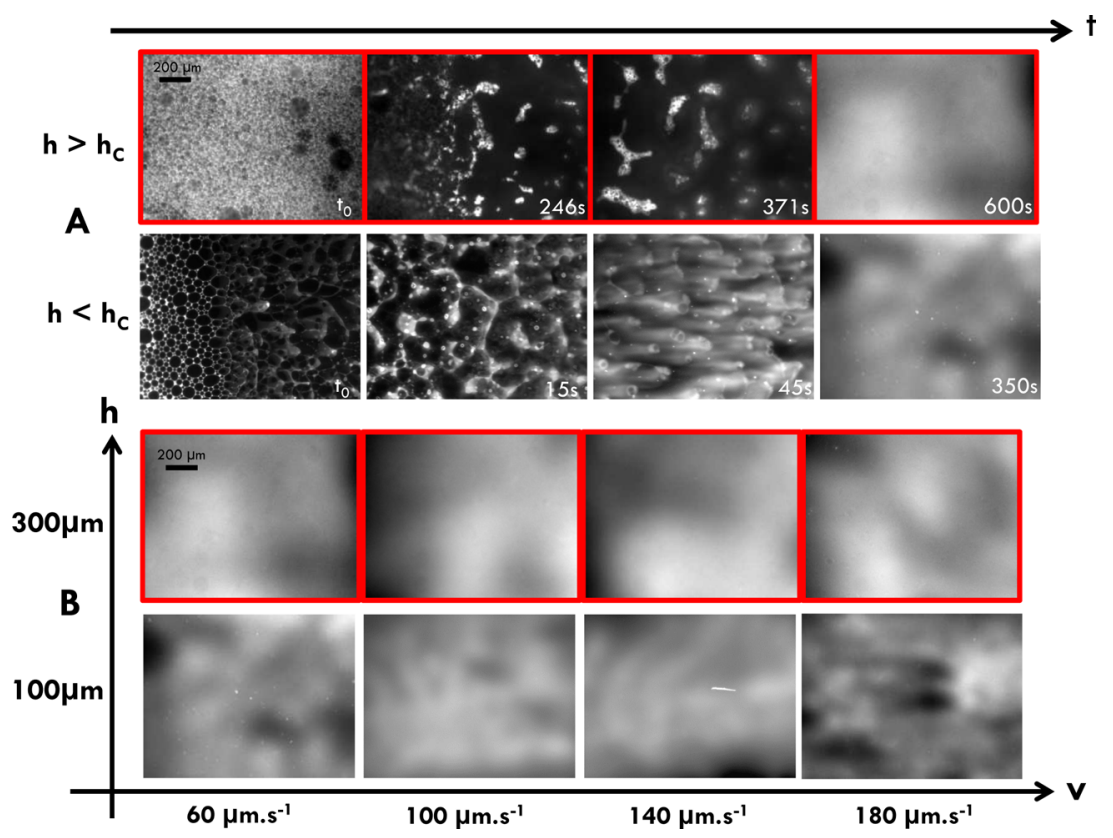

Figure 7. Deposits created by the spreading of the castor oil emulsion. Several photographs are shown for different heights $h$ and different translation velocities. Panel A shows the temporal evolution of the pattern at two different heights $\left(h<h_{\mathrm{c}}\right.$ and a velocity of $70 \mu \mathrm{m} / \mathrm{s}$ and $h<h_{\mathrm{c}}$ and a velocity of $60 \mu \mathrm{m} / \mathrm{s}$ ). Note the presence of a drying front propagating toward the blade (to the left of the image) in the second image from the left for $h>h_{\mathrm{c}}$. In panel B we show patterns after a long waiting period in fluorescence for different velocities and two different heights: a roughly homogeneous layer of water ends up coating the substrate, but variations in thickness (fluorescence intensity) are still visible.

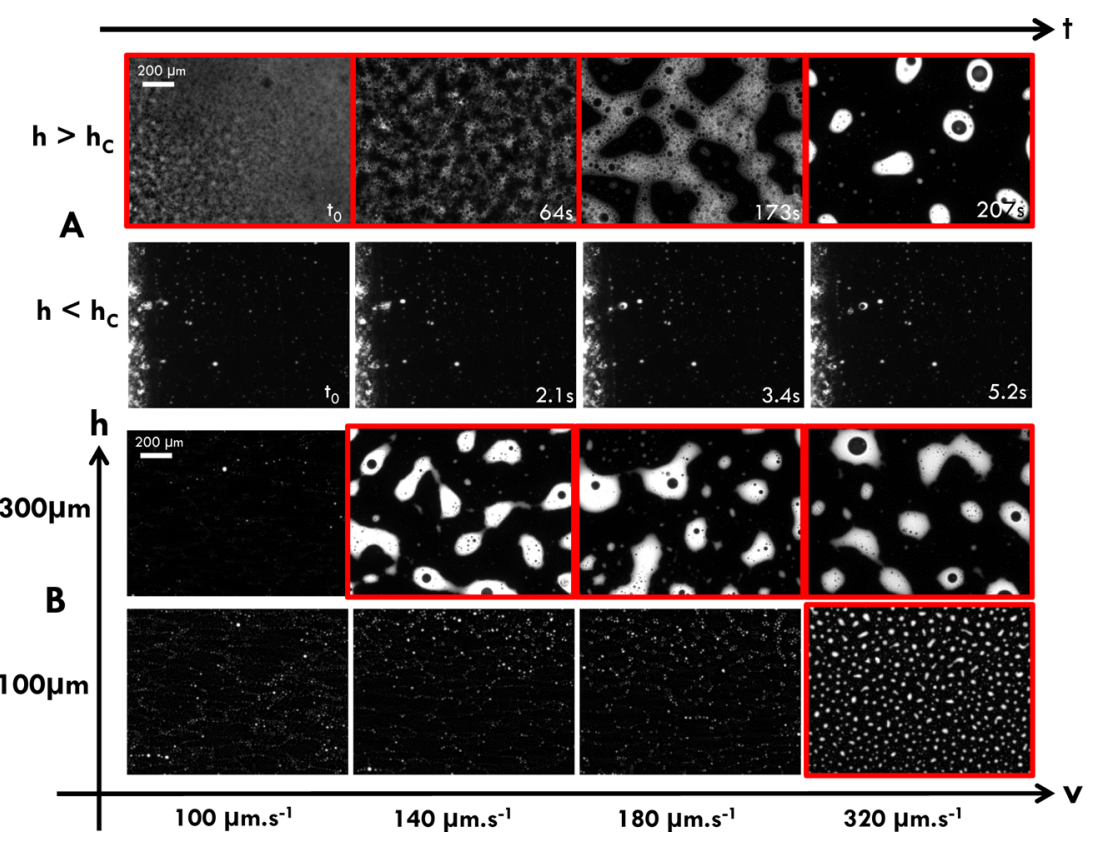

Figure 8. Deposits created by the spreading of the silicone oil emulsion. Several photographs are shown for different heights $h$ and different translation velocities. Panel A shows the temporal evolution of the pattern at two different heights $\left(h<h_{\mathrm{c}}\right.$ and a velocity of $20 \mu \mathrm{m} / \mathrm{s}$ and $h>h_{\mathrm{c}}$ and a velocity of $120 \mu \mathrm{m} / \mathrm{s}$ ). Note the presence of a nucleation and growth phase for $h>h_{\mathrm{c}}$. Panel B shows patterns after a long waiting period in fluorescence for two different heights and different velocities: drops of the continuous phase (bright) are observed which contain oil drops for the large ones, i.e., multiple emulsion drops are obtained after the inversion.

homogeneous layer of the aqueous phase as shown in Figure 7B. One can still notice variations in thickness of this layer because the fluorescence is not homogeneous throughout.

For the silicone oil emulsion, the patterns are different and depend on the translation velocity as well as on the presence of glycerine in the continuous phase. As mentioned above, low velocities give rise to a pattern of drops deposited randomly either on the bare substrate or in an oil film as shown in Figures $4 c, d$ and $8 \mathrm{~A}$. As the velocity increases, larger and larger patches of emulsion start detaching from the meniscus region, leading to the formation of an oil film containing multiple drops: water drops (bright in the images of Figure 8) with small oil drops inside (dark in the images of Figure 8). The water drops generally grow in size as the velocity increases or as the height 

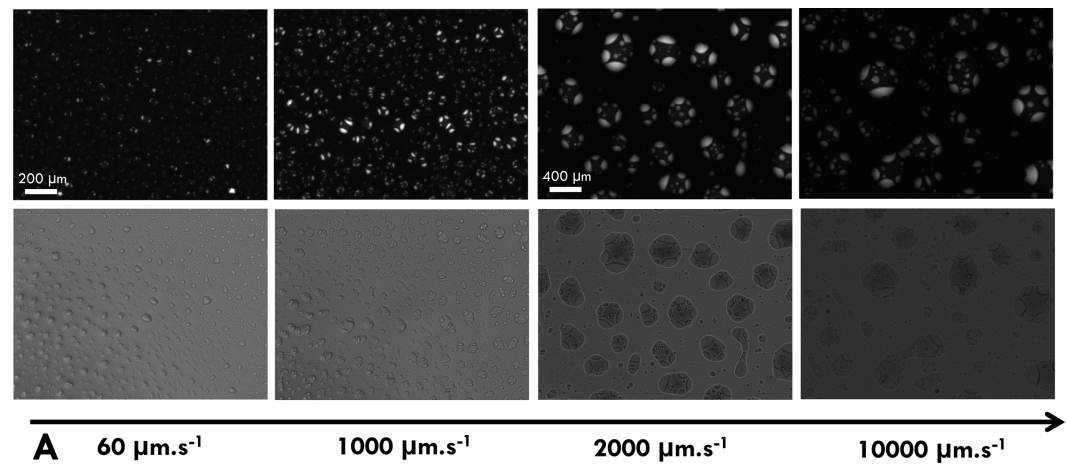

$1000 \mu \mathrm{m} . \mathrm{s}^{-1}$

$2000 \mu \mathrm{m} \cdot \mathrm{s}^{-1}$
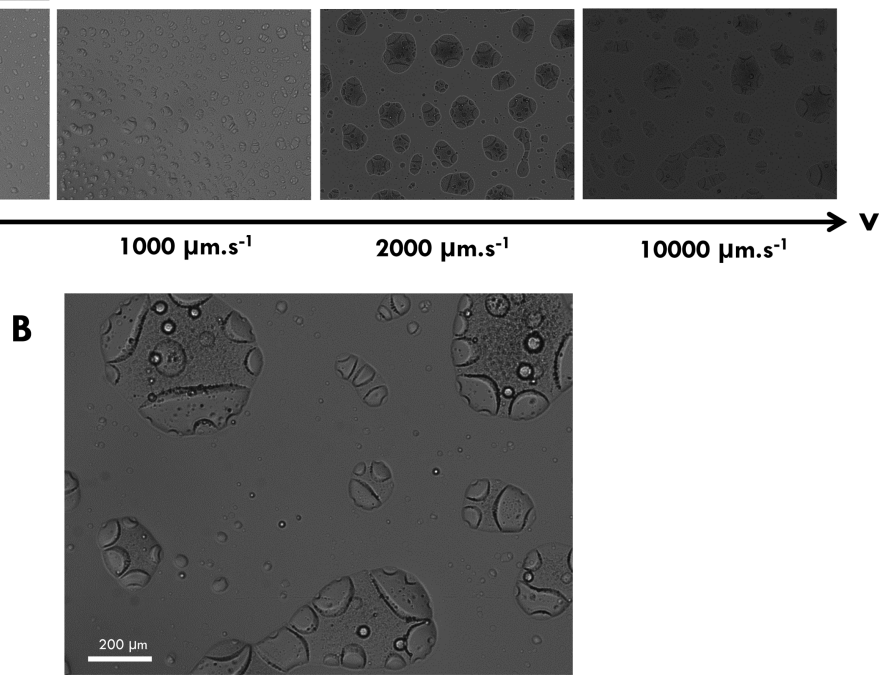

Figure 9. Deposits created by the spreading of a silicone oil in water emulsion with glycerol (water/glycerol 50\% and concentration of surfactant NP7 20\%) for different velocities. Note the phase separation within the droplets and the different patterns left behind as the soap or glycerol concentration changes.

of the blade increases. Large water drops containing several oil drops can be seen in the photographs of Figure 8. Although no time evolution is seen for the deposits at heights smaller than $h_{\mathcal{c}}$ temporal evolution is clearly visible for heights greater than $h_{\mathrm{c}}$ for which the layer becomes unstable through a nucleation and growth phase (Figure 8A), leading to the gradual formation of emulsion patches which then give rise to drops of the continuous phase with or without oil drops inside. The formation of multiple emulsions is common upon catastrophic emulsion inversion. ${ }^{30}$

These patterns change when glycerol is added to the continuous phase. Instead of oil drops inside large water drops, a complex pattern of water drops can be obtained. Photographs of these patterns are shown in Figure 9, where images taken in fluorescence and in bright field are shown. Note that the drops have an additional structure whose shapes and sizes vary. The drops of the continuous phase seem to be made up of two different domains: a bright one and a less bright one. In fact, the continuous phase with added glycerine phase separates into a phase rich in surfactant and containing most of the fluorescent dye and a phase poor in surfactant and containing a smaller amount of dye. Although this phase separation is barely visible in the stable emulsion, once demixing occurs, phase separation occurs rapidly within the drop of the continuous phase. Different patterns can be obtained either as small drops within larger ones or as more elongated domains. Note also that this pattern is nontrivial because the surfactant layers need to adopt specific configurations for such patterns to exist. For example, when a surfactant rich patch is adjacent to a water patch, a bilayer of surfactant separates the two phases. However, when oil is adjacent to water or the surfactant rich phase, a monolayer of surfactant separates the two domains. The bilayers of surfactant are more stable in the presence of glycerine and for large amounts of soap because these patterns are observed only at high soap concentrations (20\%) and for large quantities of glycerol (50\%).

\section{DISCUSSION}

Spreading an emulsion on a solid surface using a rigid blade turns out to be a complex process leading to a variety of situations. First, the transition from partial wetting to homogeneous emulsion film deposition is different from its Newtonian counterpart and seems to depend on the fact that these fluids possess a yield stress. A redefinition of the capillary number to take into account this aspect seems to give a reasonable criterion for this transition to occur. Previous work has already pointed out the importance of the yield stress in setting the thickness of yield stress fluids in dip coating experiments. $^{10}$

Perhaps the most striking aspect of these experiments is the complex nature of the contact line between the emulsion and the substrate: the destabilization and eventual inversion of the emulsion in the meniscus region with the solid surface seems to be common to both systems studied here. This inversion follows well defined dynamics for the drop size increase before inversion occurs following coalescence between drops in the contact line region. Our analysis suggests that the dynamics may be viewed as singular when the drop size increase is measured with respect to the time the inversion occurs. Our analysis seems to be consistent with previous results suggesting the existence of a constant coalescence frequency per unit surface. It is possible that a modification of this scaling using a constant coalescence frequency per unit length is more relevant to later times when the drops are flattened in a thin layer.

The inversion of the emulsion is also very sensitive to the wetting properties of the constituents of the emulsion with the solid substrate. To that end, we have tested two different systems where in the first one the continuous aqueous phase is favored on the substrate whereas in the second it is the 
dispersed oil phase which is favored. In the first case, the printed pattern after the inversion is that of a continuous and interconnected network of water ligaments on the surface. In the second system, water drops or assemblies of drops are printed on the substrate.

Finally, and for sufficiently large heights or sufficiently large velocities, a homogeneous film is deposited. The destabilization of the emulsion film under the action of evaporation of the continuous aqueous phase occurs via a drying front propagating toward the blade in one case or through a nucleation and growth mechanism in the second case. In previous work it was found that drying stress may lead to either destabilization mechanism depending on the critical pressure necessary to destabilize the emulsion. High critical pressures favor a drying front whereas low critical pressures favor nucleation and growth in the bulk of the emulsion. ${ }^{29}$ In our case we believe that the differences in wetting properties of the dispersed phase may also be responsible for the differences between the two systems because in the silicone oil emulsion the oil is favored at the substrate and coalescence of the drops with the glass may favor nucleation. For the castor oil system it is the continuous phase which is favored at the glass surface, and coalescence occurs near the front where evaporation is strong. The patterns obtained after the destabilization on the surface are also dependent on the emulsion properties. Interconnected net works or multiple and complex drop patterns can be obtained. Perhaps the most striking pattern is obtained when glycerine is included in the aqueous phase. Here complex drops of water and soap rich regions are dispersed in oil. As the continuous phase undergoes phase separation, additional structure within the drops can be obtained. Such additional structure can be useful for making Janus like particles, as has been shown recently. ${ }^{31}$

\section{CONCLUSIONS}

This study shows the rich phenomenology of a simple act: spreading an emulsion on a plate using a blade. Different phenomena occur, namely, emulsion inversion, coalescence and droplet growth, complex pattern formation, and eventual deposition of a thick emulsion film under relatively well defined conditions. We have put forth these different cases by doing a systematic study of the coating process at different speeds and different blade heights and by using two different emulsion systems. The relative wetting of the dispersed and continuous phases was shown to have an important impact on the emulsion destabilization and inversion as well as on the deposited patterns. The velocity of the deposition process was also shown to influence the end result through a transition to a thick film as expected for Newtonian fluids, but here the yield stress and the shear thinning character of the fluids have to be taken into account. Not only is the non Newtonian character of the fluid important, but the fact that it is a two fluid dispersion turns out to be crucial.

\section{AUTHOR INFORMATION}

\section{Notes}

The authors declare no competing financial interest.

\section{ACKNOWLEDGMENTS}

We acknowledge LabEx AMADEus, ANR 10 LABX 0042 AMADEUS, and the program of Excellence Initiative IdEx
Bordeaux (grant no. ANR 10 IDEX 0003 02) for financial support. H.K. acknowledges support from IUF.

\section{REFERENCES}

(1) Weinstein, S. J.; Ruschak, K. J. Coating Flows. Annu. Rev. Fluid Mech. 2004, 36, 29-53.

(2) Corvolan, C. M.; Saita, F. A. Blade coating on acompressible substrate. Chem. Eng. Sci. 1995, 50, 1769-1783.

(3) Saita, F. A. Simplified models of flexible blade coating. Chem. Eng. Sci. 1989, 44, 817-825.

(4) Iliopoulos, I.; Scriven, L. E. A blade coatingstudy using a finite element simulation. Phys. Fluids 2005, 17, 127101.

(5) Eklund, D. E. Influence of blade geometry and blade pressure on the appearance of a coated surface. In Proceedings of the 1984 TAPPI Coating Conference; 1984; pp 37-43.

(6) Kistler, S. F.; Schweizer, P. M.; Laurence, R. L., Middleman, S. Liquid Film Coating; Chapman \& Hall,1997.

(7) Hsu, Tong C.; Malone, M.; Laurence, R. L.; Middleman, S. Separating forces in blade coating of viscous and viscoelasticliquids. JNNFM 1985, 18, 273-294.

(8) Ross, A. B.; Wilson, S. K.; Duffy, B. R. Blade coating of a power law fluid. Phys. Fluids 1999, 11, 958.

(9) Seiwert, J.; Quere, D.; Clanet, C. Flexible scraping of viscous fluids. J. Fluid Mech. 2013, 715, 424-435.

(10) Maillard, M.; Boujlel, J.; Coussot, P. Solid Solid Transition in Landau Levich Flow with Soft Jammed Systems. Phys. Rev. Lett. 2014, 112, 068304.

(11) Bretherton, F. P. The motion of long bubbles intubes. J. Fluid Mech. 1961, 10, 166-168.

(12) Derjaguin, B. On the thickness of the liquid film adhering to the walls of a vessel after emptying. Acta Physicochim. USSR 1943, 20, 349-352.

(13) Landau, L.; Levich, B. Dragging of a liquid by amoving plate. Acta Physicochim. USSR 1942, 17, 42-54.

(14) Delon, G.; Fermigier, M.; Snoeijer, J. H.; Andreotti, B. Relaxation of a dewetting contact line. Part 2 Experiments. J. Fluid Mech. 2008, 55, 604.

(15) Snoeijer, J. H.; Andreotti, B. Moving ContactLines: Scales, Regimes, and Dynamical Transitions. Annu. Rev. Fluids Mech. 2013, 45, 269.

(16) Eggers, J. Contact line motion for partially wetting fluids. Phys. Rev. E 2005, 72, 061605.

(17) Eggers, J. Existence of receding and advancing contactlines. Phys. Fluids 2005, 17, 082106.

(18) Bonn, D.; Eggers, J.; Indekeu, J.; Meunier, J.; Rolley, E. Wetting and spreading. Rev. Mod. Phys. 2009, 81, 739.

(19) Leal Calderon, F.; Schmitt, V.; Bibette, J. Emulsion Science, Basic Principles, 2nd ed.; Springer: New York, 2007.

(20) Wasan, D. T.; Nikolov, A. D.; Aimetti, F. Texture andstability of emulsions and suspensions: role of oscillatory structural forces. Adv. Colloid Interface Sci. 2004, 187-195.

(21) Bremond, N.; Doméjean, H.; Bibette, J. Propagation of Drop Coalescence in a Two dimensional Emulsion: A route towards Phase Inversion. Phys. Rev. Lett. 2011, 106, 214502.

(22) Brailsford, A. D.; Wynblatt, P. The Dependence ofOstwald Ripening Kinetics on Particle Volume Fraction. Acta Metall. 1978, 27, 489-497.

(23) Yao, J. H.; Elder, K. R.; Guo, H.; Grant, M. Ostwald Ripening in Two and Three dimensions. Phys. Rev. B 1992, 45, 8173-8176.

(24) Paredes, J.; Michels, M. A. J.; Bonn, D. Rheology across the Zero Temperature Jamming Transition. Phys. Rev. Lett. 2013, 111, 015701.

(25) Huang, J. R; Mason, T. G. Shear oscillation lightscattering of droplet deformation and reconfiguration in concentratedemulsions. EPL 2008, 83, 28004.

(26) Kao, J. C. T.; Blakemore, A. L.; Hosoi, A. E. Pullingbubbles from a bath. Phys. Fluids 2010, 22, 061705.

(27) Goyon, J.; Colin, A.; Ovarlez, G.; Ajdari, A.; Bocquet, L. Spatial cooperativity in soft glassy flows. Nature 2008, 454, 84-87. 
(28) Deminiere, B.; Colin, A.; Leal Calderon, F.; Muzy, J. F.; Bibette, J. PRL 1999, 82, 229-232.

(29) Feng, H.; Sprakel, J.; Ershov, D.; Krebs, T.; Cohen Stuart, M. C.; van der Gucht, J. Two modes of phase inversion ina drying emulsion. Soft Matter 2013, 9, 2810-2815.

(30) Preziosi, V.; Perazzo, A.; Caserta, S.; Tomaiuolo, G.; Guido, S. Phase Inversion Emulsification. Chem. Eng. Trans. 2013, 32, 15851590.

(31) Chen, C. H.; Shah, R. K.; Abate, A. R.; Weitz, D. JanusParticles Templated from Double Emulsion Droplets Generated UsingMicro fluidics. Langmuir 2009, 25, 4320-4323. 